\title{
THE CAUSES OF ATMOSPHERIC INHOMOGENEITIES
}

\author{
R. S. SGORER.
}

Summary. - There are several different atmospheric causes, of imperfections in the images seen in a telescope. The most serious are due to thermal convection from the ground, or the stirring of a stable layer at the ground by wind : choice of site and observatory design can reduce these effects. In the free air well away from the ground some of the largest inhomogeneities may be due essentially to large gradients of humidity, and certain cloud types indicate the mechanisms by which such inhomogeneities are established. Temperature fluctuations caused by pressure fluctuations in eddies close to the telescope are probably not serious, and can be avoided by observatory design.

1. The problems to be considered. - We shall not be concerned with cloudiness or atmospheric turbidity in this paper. The most serious imperfections in images are due to inhomogeneities of refractive index whose length scale is comparable with the telescope diameter. Except close to the ground they are on a scale which is much smaller than can be detected by ordinary radio-sonde balloons, and only recently have instruments been fitted to aircraft which can detect fluctuations on a length scale of less than a metre. Close to the ground we have a fairly clear idea of what causes the inhomogeneities; but in the free air, above $\mathrm{I} \mathrm{km}$ from the ground we depend mostly on inference from what we can see in cloud forms and from measurements by aircraft on a larger scale than a metre or two : there is still very little direct evidence such as we have in abundance for larger scales of motion. Consequently a proper understanding of the mechanics of motion in the free atmosphere is necessary if the inferences are to be reliable.

2. Temperature fluctuations caused by the stirring of stable layers. - (i) Stable layers at the ground. - When the ground cools at night it acquires a much lower temperature than the air above it. The air becomes stably stratified; when disturbed, parcels of air tend to return to their former level. The stability is measured by the quantity

$$
g \beta=g \frac{1}{\theta} \frac{\partial \theta}{\partial z},
$$


where $g$ is gravity, and $\theta$ is the potential temperature (for definition see, for example, Scorer, I958, p. I69-172) whose reciprocal plays the same role in the atmosphere as density does in a stratified incompressible liquid. Thus in the case of water of salinity (and therefore of density) varying with height, the stability would be represented by

$$
g \beta=-g \frac{1}{\rho} \frac{\partial p}{\partial z}
$$

Stirring motions, which would carry parcels of air from one level to another (fig. 24), and thereby cause fluctuations of temperature, are produced when the orderly shearing motion, due to the wind being weaker at the ground than higher up, is made disorderly by the passage of the air over obstacles or, in extreme cases, by the instability of large shear.

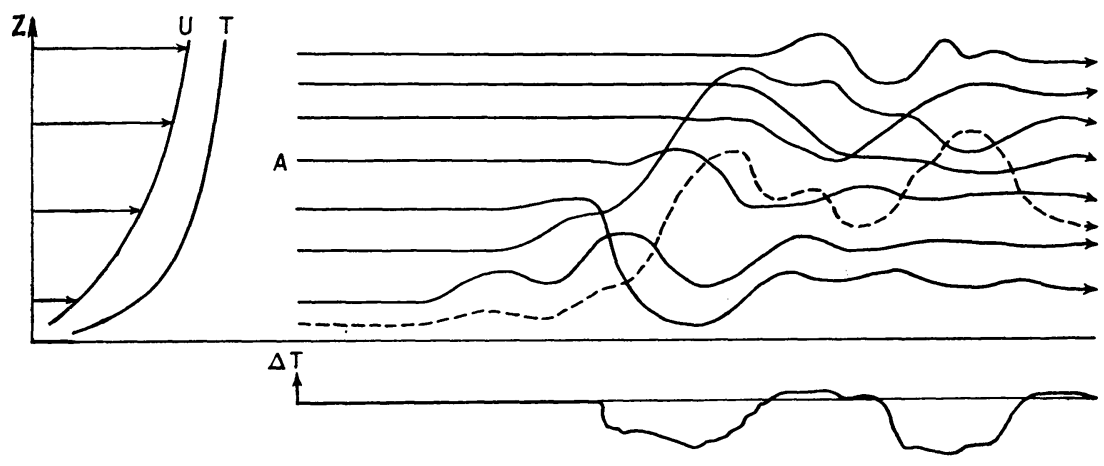

Fig. 24 .

On left : Velocity and temperature profiles close to the ground when the ground is cooled at night; $U$, velocity; $T$, temperature; $Z$, height.

On right : Sample tracks of air particles in unsteady motion, illustrating how the displacement from their original level produces temperature fluctuations at the fixed height $\mathrm{A}$.

The stirring motions thus derive their energy from the shear of the mean wind, which is represented by

$$
\mathbf{U}^{\prime}=\frac{\partial \mathrm{U}}{\partial z}
$$

The Richardson number is the non-dimensional number which represents the ratio of the stabilising and stirring influences, and is given by

$$
\mathrm{R}_{i}=\frac{g \beta}{\mathrm{U}^{\prime 2}} .
$$


Much is made of this number in the literature, and it is known that stirring motions are more likely to occur when this number is small than when it is large. But the precise value of $R_{i}$ at which smooth (laminar) flow will be replaced by stirred flow depends very much on the roughness of the ground and the height above the ground.

The most useful advice about observatory sites is that obstacles to smooth flow will cause the air to be stirred, and therefore, if the surface is flat, it should be as smooth as possible upwind of the telescope.

Isolated mountain peaks with precipitous upwind slopes are often immersed in air that has arrived horizontally across a valley, and the stable stratification at night is therefore much less than in air which has been close to cold ground.

The peak itself will be cold, and will therefore have a stable layer close to its surface, but this layer may be very shallow (less than I $\mathrm{m}$ ) near to the upwind edge of the peak and it may be easy to place the telescope above it. Cooling of the dome may produce similar fluctuations close to the telescope, and this problem is discussed in section 5 .

A parcel of air carried from close to the ground to the top of the telescope may be surrounded by air whose temperature is of the order of $\mathrm{r}^{\circ} \mathrm{C}$ warmer. This will produce serious image distorsions. The buoyancy forces will tend to force such a parcel downwards again and the higher the parcel is displaced from near to the ground the more it will have become mixed on the way; the magnitude of the temperature fluctuations therefore decreases upwards.

The breakdown of smooth into stirred flow over level ground has been long studied (see Brunt, 1939, p. 238 et seq). On mountain top sites, a local study with thermometers sensitive to fluctuations of period $0.3 \mathrm{~s}$ or so would reveal their typical characteristics. Most useful would be a survey with a quickly moved thermometer, sampling several positions at various distances from the upwind edge of the site, to see how the stirred layer grows in thickness from the upwind edge (fig. 25).

(ii) Stable layers in the free atmosphere. - There are various mechanisms whereby a stable layer in the atmosphere at a height of a few kilometres can be stirred while the air above and below remains in laminar motion. They are discussed at length by Scorer ( 1963 ) in connection with the problem of bumpiness experienced by aircraft in clear air far from the ground. It is clear, at least, both from theory and observation, that the presence of a mountain beneath a smoothly flowing airstream can produce stirring in fairly thin layers far above the ground. The interesting point is that the most stable layers (largest $\beta$ ) are often the most likely to be stirred, and so temperature fluctuations are very readily produced. Nothing can be done about these by choice of site within a few miles, or by observatory design. The wave motions in 
which this stirring occurs most often, are commonest where there is a strong wind at high levels in the same direction as the wind near the mountain surface. This situation occurs in a jet-stream associated with the polar front, but is less common in the subtropical jet-stream because the winds are weaker and are not so often associated with winds in the same direction at the ground. A jet-stream itself is not a turbulent phenomenon; but, in the case of the polar front jet-stream, the stirring is often severe in it when it contains very stable layers and passes across mountain ranges. It will be seen in section 4 that these stirring motions may cause more trouble on account of the fluctuations of humidity which they produce.

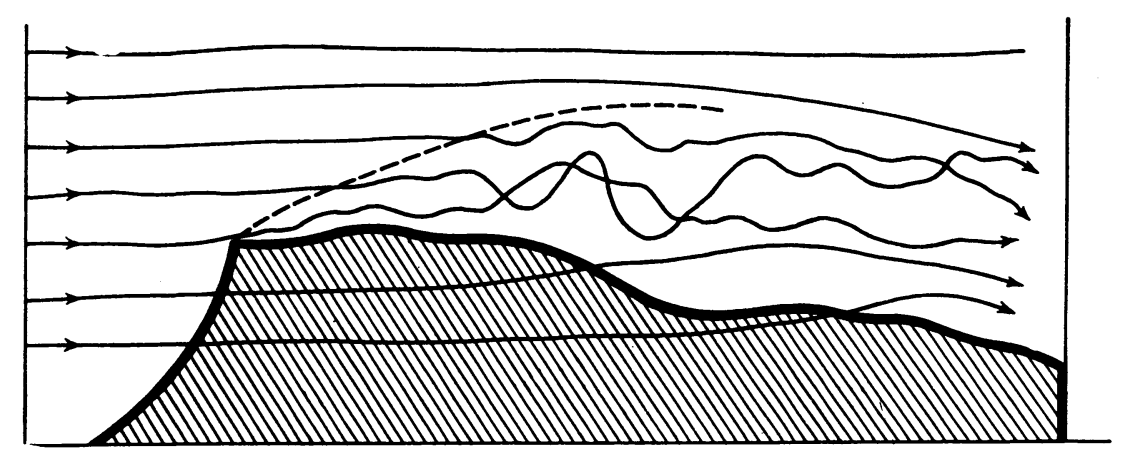

Fig. 25. - The stirred region of a stable airstream grows in depth downwind from the front-edge of a mountain. The usual extent of this region can only be found reliably by means of local surveys.

3. Temperature fluctuations in thermal convection. - When the ground is much hotter than the air above no wind shear is necessary to start stirring motions because the air is unstably stratified. Theoretical treatments deal with thermal convection over a smooth uniform surface, but close to the ground where the temperature fluctuations are a nuisance to observers of the Sun, the detailed nature of the surface can have a large effect. It is well known to glider pilots that certain kinds of surface are good thermal sources, and send up many more thermals (i. e. bodies of buoyant air rising through their surroundings) than the ground round about them. If a deliberate attempt is to be made to prevent thermals being produced close to an observatory the greatest effect is obtained by having green, growing vegetation in well watered ground. In this way a very large fraction of the insolation that is absorbed is converted into latent heat by the evaporation of water through the vegetation, and the temperature is kept much lower than over bare ground with the same radiative properties. 
A snow surface just above the snow line is also almost free from convection because : $(a)$ it reflects sunshine very efficiently, $(b)$ it cannot be raised to above $o^{\circ} \mathrm{C}$, and is usually kept at a lower temperature by evaporation of ice, $(c)$ it radiates efficiently as a black body in the infrared. Snow therefore produces a kind of anti-greenhouse effect and keeps the surface at a nearly constant cold temperature in sunshine.

This effect of snow is very noticeable when the air is stably stratified : anabatic (upslope) winds, usually less than roo $\mathrm{m}$ thick, blow up hillsides as far as the snow-line but no further. A haze top, indicating the upper limit of convection is often seen at the snow line.

A promontory on a hillside may often protrude to near the top of the anabatic current, and in this way may be outside the layer in which the temperature fluctuations are greatest (close to the warmed hillside). This will be particularly so if the air away from the hillside is stably stratified; but even if it is not, and the convection is not confined to a shallow layer close to the surface but extends up to several hundreds of meters, if there is a wind a small promontory or hillock will be above the layer of greatest temperature fluctuations. If, however, there is not normally much wind, the hillock may act as a point of convergence for thermals and it would therefore be subject to greater temperature fluctuations than the flat ground nearby.

The way in which magnitude of the temperature fluctuations varies with height may be very roughly deduced as follows. Over uniform ground the only quantity which exists to determine the size of thermals is distance above the ground. The upward velocity $w$, is dependent only on the size and the density deficiency $\Delta \rho$, and is shown by dimensional arguments to be given by

$$
w \propto\left(g \frac{\Delta \rho}{\rho} z\right)^{\frac{1}{2}} \propto\left(g \frac{\Delta \theta}{\theta} z\right)^{\frac{1}{2}}
$$

If the upward flux of heat is the same at all heights then

$$
w \Delta \theta=\text { constant (independent of } z \text { ) }
$$

and so

$$
\Delta \theta \propto z^{-\frac{1}{3}}
$$

and

$$
z^{-1} \Delta \theta \propto z^{-\frac{4}{3}}
$$

This last quantity is a measure of the nuisance value of a thermal to a telescope, and the advantages of getting as high above the ground as possible are obvious. 
Clearly (3.4) cannot hold right down to the ground. Within a few centimetres molecular thermal conduction is important, but also, and more important, there is a considerable transfer of heat by radiative exchange in the infrared between the ground and the water vapour in the air. This is important in ordinary humidities up to a height of about $2 \mathrm{~m}$, but may have an influence up to $10 \mathrm{~m}$ in very dry air. Beyond that height there is some upward heat flux due to re-emission and absorption by the water vapour higher up, but this is not important in comparison with the transfer by convection. The existence of this heat flux by infrared radiation in the lowest few metres reduces the flux by convection and therefore reduces $\Delta \theta$ by comparison with the value predicted in (3.4). A dry atmosphere would therefore appear to offer an advantage by reducing the temperature fluctuations in thermal convection in a deeper layer of air (by transferring the heat by another mechanism). But this effect is far outweighted by the reduction of the temperature fluctuations by having a large evaporation of water from the surface.

It is dangerous to generalise about the behaviour of winds in valleys and near mountain sides. Different sites may have markedly different properties because of local topographic features. In stable air anabatic winds are shallow, but if the whole depth of the valley air mass is subject to warming by the penetration of thermals it may all be set in motion as a valley wind of some sort. On the other hand, winds in long valleys may often blow along the valley towards the end at which low pressure has been established by a much larger scale weather system; and in this case the diurnal variations may be small.

Catabatic (downslope, nocturnal) winds are usually shallow. An observatory on a hillside might be disturbed by them by the stirring of the air close to the cooled surface : again a suitable promontory on a mountain side might reach above these shallow winds. It is also possible that the air may accumulate as a cold pool in the valley bottom and be horizontally stratified and so cause no seeing troubles. But to choose a valley bottom site for nocturnal observation would usually be rash on this argument alone : winds blowing across the top will often produce stirring of stable layers in the free air, and sometimes regular gusts of wind are set up by the periodic penetration of higher level air into the valley.

4. Effects of fluctuations of humidity. - Variations of humidity, more than fluctuations of temperature, are responsible for the variations of the refractive index of air for radar wavelengths. In the case of optical wavelengths this effect is negligible but a more subtle effect replaces it. 
Water vapour has a density which is 0,622 times that of dry air. As a consequence, if two neighbouring parcels of air have the same density, but different humidities, they must have different temperatures. The order of magnitude of this effect is given with sufficient accuracy as

(4.1) $0 . \mathrm{I}^{\circ} \mathrm{C}$ per gramme of water vapour per kilogramme of air.

Thus if a parcel of air contains $\mathrm{I} g$ of vapour per kilogramme less than a neighbouring parcel but is at the same density its temperature must be $0.1^{\circ} \mathrm{C}$ warmer.

This fact is important because when the density is uniform along a horizontal surface stirring motions tend to die out. Consequently disturbances which mix masses of air of different humidities and die out again when the density is uniform can leave a patchy distribution of humidity which is only finally smoothed out by molecular diffusion over many hours. The table below shows the amount of water vapour per kilogramme of air at various temperatures and pressures when the air is saturated. If these amounts, which are given in grammes are divided by Io we have the maximum temperature difference in degrees centigrades which can result from variations in humidity at constant density.

TABLE I.

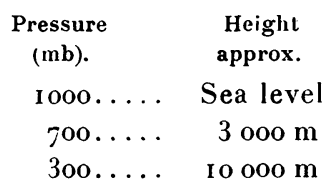

\begin{tabular}{rccccc}
\multicolumn{7}{c}{ Grammes } & $\mathrm{H}_{2} \mathrm{O}$ per kilogramme air. \\
\hline 1. & 2. & 4. & 8. & 16 & 32. \\
-17 & -8.5 & -0.5 & 10.5 & $2 \mathrm{I}$ & 32.5 \\
-21 & -13 & -4 & 3.5 & 13.5 & 24 \\
-30.5 & -23 & -15 & -6 & - & -
\end{tabular}

Table I gives the temperatures in degrees centigrades at which the air is saturated bythe amount of water vapour shown, at the given pressures. At these temperatures and pressures the maximum temperature anomaly due to humidity variations is in degrees centigrades about $\mathrm{r} / \mathrm{roth}$ of the mass of water vapour shown in grammes. Thus at $700 \mathrm{mb}$ and $3.5^{\circ} \mathrm{C}$ the maximum effect is $0.8^{\circ} \mathrm{C}$.

Near the ground the magnitude of the effect under consideration is small compared with the fluctuations of temperature of the air if only because large variations of humidity are not normally maintained there; but in the free air it may be the major effect, partly because the surfaces of constant humidity may be inclined very steeply to the horizontal so as to produce substantial image movement as they pass even when the length scale of the inhomogeneities is fairly large compared with the telescope diameter. The rays may intersect the boundaries of fairly large inhomogeneities tangentially and be deflected after the 
manner of a mirage. The next section describes some of the cloud forms which can easily be observed which accompany the mechanisms which produce the humidity inhomogeneities.

5. Mechanisms producing static humidity variations. - By static humidity variations we mean variations which are built into the air and which do not disappear when the motion which produced them vanishes. One of the most striking pieces of evidence for the existence of these variations is in the sizes of the cloudlets which appear typically ahead of warm fronts. When a layer of cloud is formed by the widespread upward motion of the air the size of the cloudlets appears to grow as the cloud thickens. This is not because the cloudlets are the product of a motion which increases in scale as the cloud layer thickens, for the larger cloudlets are already apparent in the pattern of smaller ones which occurred earlier; moreover there is usually no internal motion visible in the clouds, and any there may be is ineffective compared with the changes in cloud appearance due to the thickening of the layer. The small cloudlets which appear first are the regions of greatest humidity, and are usually in the form of knobbles on the upper surface of the larger cloudlets which appear later. Plate $\mathrm{V} a$ is an example of the edge of such a cloud layer.

(i) Fallstreaks. - Plate $\mathrm{V} b$ illustrates fallstreaks, which are streamers of ice crystals falling from a cloud which is usually, but not always, a convection cloud. The streamers do not normally reach the ground : they evaporate, producing a column of air which is more humid than the environment.

(ii) Cumulus. - Ordinary fairweather cumulus (plate VI $a$ ) are composed of thermals. The smaller clouds consist of only one thermal each which evaporates before a second rises into the same region of air : the larger ones consist of several thermals, and since the evaporation which is taking place over the outside is, for a time, offset by the passage of new thermals through the base, the clouds grow taller. Each cumulus, after it has evaporated, leaves a dome-shaped region in which the humidity is greater than in the surroundings.

(iii) Castellanus. - These are turret-like cumulus (plate VI b) which sprout from a thin strip or layer of cloud. They differ from ordinary cumulus in that they obtain their buoyancy entirely from the condensation of cloud and not from the heating of the ground. They often rise from very thin layers of high humidity into very dry layers, and as they evaporate they produce a very patchy distribution of humidity. The smallest cloudlets may be only a very few metres across. No study has been made of the smallest knobbles on this or any other type of cumulus. 
(iv) Alto-cumulus. - By this name (alto-) we mean a cloud formed away from the direct influence of the ground but whose shape is the result of the cumulus (convection) type of motion. (Castellanus is a special type of altocumulus which sprouts up into the air above.) If a thin layer of cloud is formed, with clear sky above, the loss of heat by long wave radiation from the top and the absorption of it from the ground below produces static instability. The layer then breaks up into cloudlets, often with clear spaces in between. If there is wind shear the cloudlets are arranged in rolls across the shear (plate VII $a$ ).

If a layer as a whole undergoes down and up motion the cloud disappears and reappears with the same pattern of cloudlets. Often such cloud is produced in the first place in the crest of a wave over or near to a mountain, and disappears from the air as it passes the mountain. A mottled pattern of humidity is then built into the air (plate VII $b$ ).

(v) Billows. - An example of billows is illustrated in plate VII $a$. In that case the instability was primarily due to cooling of the top and heating of the bottom of the cloud layer, but if the shear is large enough a pattern of rolls is produced in the air even though it remains statically stable $\left(\beta>0\right.$ and $R_{i}>0$ ). Examples of clouds revealing this type of motion have been given by Scorer (1963). Most visible billows are of a length scale of several tens of metres, but occasionally much smaller. The billow motion can corrugate and "crumple" thin layers of air of high humidity, whether they contain cloud or not, and thereby produce an uneven distribution of humidity.

(vi) Thin humid layers. - The atmosphere is so often subjected to stirring motion by thermal convection and rain-producing systems that it is not generally appreciated how readily thin humid layers interleaved with much drier layers can be produced. Plate VIII shows how thin layers can sometimes become visible as laminations in a wave cloud. It is most probable that these thin layers are produced by the shearing over of towers of higher humidity produced by convection clouds. Isolated thunderstorms can produce great towers of moister air a few miles in width; and when the air into which they were evaporated has been sheared over, within a day or two the top of the tower may be several hundred miles from the bottom and the humid air extended into a slightly inclined sheet only a few metres thick. Smaller towers such as those produced by castellanus clouds can be distorted into streaks of damper air only a few centimetres thick. These in turn may become sources of small castellanus clouds when they are condensed again into cloud by ascending motion over a hill or in some other way.

Thin humid streaks may also be produced by the evaporation of fallstreaks into a shearing airstream. 
The important feature of the atmosphere which makes this possible is the smooth shearing motion which. can continue for days at a time without any stirring motion taking place. If however a thin damp layer is stirred into the air above and/or below it, the ultimate state can easily contain large horizontal gradients of humidity, and therefore of temperature, if these were, before the stirring began, large vertical gradients and thin humid layers.

6. Eddies due to wind blowing past an observatory. - We come now to the question " can the eddying motion due to obstacles in an airstream of uniform potential temperature cause temperature fluctuations which produce significant image distortion ?" The maximum effect is not likely to exceed the dynamic temperature rise which occurs when the air is brought to rest, for example at the stagnation point on the front of an obstacle where the flow divides and passes round the obstacle. A pressure change of I mb produces a temperature change of approximately $0^{\circ}{ }^{\circ} \mathrm{C}$. Table II gives the dynamic pressure rise in $\mathrm{mb}$ corresponding to various wind speeds ( $1 \mathrm{~m} / \mathrm{s}=2 \mathrm{knots}$ ).

TABLE II.

\begin{tabular}{|c|c|c|}
\hline n.m. & 5 & 10 \\
\hline Pressure rise ( $\mathrm{mb})$ at stagnation point (sea level) & $\mathbf{I} / 8$ & $1 / 2$ \\
\hline
\end{tabular}

Pressure drops could exceed the pressure rises in magnitude, the most significant example being the pressure drop in the centre of a wingtip vortex on an aircraft; but unless an obstacle were designed to produce such vortices the pressure drop would in general be much less than the value given in table II.

In stationary eddies, such as might be formed in a hole made in a flat surface over which the wind was blowing, the same air circulates in the eddy with slow mixing with the surroundings. The consequence is that the temperature difference is reduced, usually, to less than a quarter of what it would be if air from the freestream could reach the centre of the eddy without mixing. In moving eddies the centrifugal forces which produce the low pressures in their centres are not as large as in more organised stationary eddies and so temperature fluctuations are probably more nearly about a tenth of those given in table II.

It is probably worth ensuring that there are no protruding features on an observatory whose wakes lie across the line of site of the telescopes, including the wake of the dome itself (see section 7) but differences of temperature between the dome and the surrounding air are much more likely to cause image distortion than these purely dynamic effects. It is therefore safe to say that any shape producing stirring motions 
should be avoided on an observatory primarily because it will stir air near a solid surface, at a different temperature from the free air, into the free air, and that the dynamic effects are relatively unimportant (unless the shape is designed to produce them).

7. Observatory design. - Above all, the design should seek to prevent air from close to the ground from passing over the top of the aperture in the roof through which the observations are being made. It is worth considering the ideal in order to see whether improvements can easily be made towards it.

The first principle should be to make the air stirred at the ground pass round or beneath the telescope. An observatory on struts is impracticable because of the need to attach the telescope to the ground by a massive pillar. But there is no need for anything other than the telescope to be supported in this way, and so the housing should be built upon struts only.

In any case the opening in the roof should be at a considerable height, say two dome diameters above the ground, and this would make it possible to build a false floor around it, as indicated in figure 26 , under which most of the stirred layer would flow. Such false floors could be made of bamboo or cloth and cheaply erected as an experiment. They could be sprayed with water in the daytime to prevent convection in the immediate neighbourhood of the observatory in sunshine, and with water at ambient air temperature to prevent excessive cooling by radiation at night to the low temperature achieved by the ground under a clear sky. A very light porous false floor, made of nylon net for

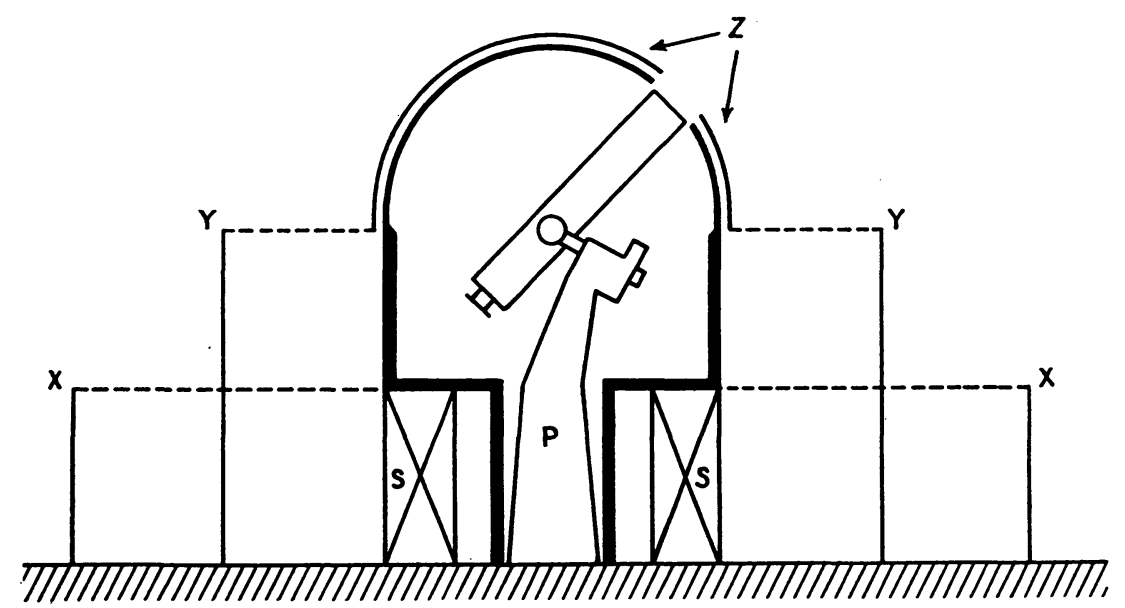

Fig. 26. - Section through proposed observatory. The heavy line shows the outline of the closed building. $\mathrm{Z}$ indicates the porous outer shell, $\mathrm{P}$ the pillar supporting the telescope, S the strutted support for the dome, XX and YY are "false floors". 
example, would greatly reduce the turbulent transfer of cold eddies over the observatory at night and would, on account of its good ventilation and small heat capacity, acquire a temperature much closer to the ambient air than the ground would.

If the dome could be streamlined some advantage would accrue at times when the line of sight were through the downwind side; but a more profitable approach would be to have a porous dome into which air was steadily sucked so as to remove the boundary layer and reduce the wake size and the intensity of eddies in it. Such a porous dome would also be held at approximately the same temperature as the outside air by which it would be ventilated so that it could not get colder than the air on a night of light wind. (It would readily be constructed of fine nylon cloth stretched over a spherical dome of wire netting and this would be built about 5-ro $\mathrm{cm}$ outside the conventional dome, air being sucked by a pump from between them.)

The edge of the window in the dome should be smooth and continuous with the rest of the surface of the dome.

A double walled tube of very porous cloth would damp out velocity fluctuations and even temperature fluctuations in air flowing through it, and could therefore be used to surround the line of sight if it lay through the wake of the dome; but it should be emphasised that this is desirable chiefly because the dome surface acquires a different temperature from the ambient air so that in the wake (where the boundary layer air flows from the surface) temperature fluctuations are produced. The false floor and suction of air through the dome surface as suggested above, are probably more important. 